\title{
White Dwarf Rotation: Observations and Theory
}

\author{
Steven D. Kawaler \\ Department of Physics and Astronomy, Iowa State University, Ames, \\ IA 50011 USA
}

\begin{abstract}
White dwarfs rotate. The angular momentum in single white dwarfs must originate early in the life of the star, but also must be modified (and perhaps severely modified) during the many stages of evolution between birth as a mainsequence star and final appearance as a white dwarf. Observational constraints on the rotation of single white dwarf stars come from traditional spectroscopy and from asteroseismology, with the latter providing hints of angular velocity with depth. Results of these observational determinations, that white dwarfs rotate with periods ranging from hours to days (or longer), tells us that the processes by which angular momentum is deposited and/or drained from the cores of AGB stars are complex. Still, one can place strong limits on these processes by considering relatively simple limiting cases for angular momentum evolution in prior stages, and on subsequent angular momentum evolution in the white dwarfs. These limiting-case constraints will be reviewed in the context of the available observations.
\end{abstract}

\section{Introduction}

Despite a flurry of interest, mainly from theorists, in the 1960s, applicable methods to observe rotation in single white dwarfs largely eluded astronomers until the 1970s. As relatively simple structures whose mechanics are dominated by the degenerate equation of state of the electrons, the influence of rotation on their structure can, in principle, be computed analytically using the theory of rotating polytropes. Rotation could provide another "force" that could support the star, increasing the maximum mass for an electron degenerate configuration. Thus the hope was, in part, that by finding white dwarfs rotating at sufficiently high velocities, physics at the interface between classical and modern could be tested. One of the earliest efforts to explore this connection was by Ian Roxburgh (Roxburgh 1965), who showed that the maximum mass of a white dwarf could be increased by about $5 \%$ if rotating at near critical velocity of over 2000 $\mathrm{km} \mathrm{s}^{-1}$. As limits on rotation velocities of single white dwarfs began to be measured in the 1970 s, it became apparent that they were rotating at much smaller velocities than that. Still, measuring the rate of rotation of white dwarfs can allow us to probe the evolution of stars in their late stages - the rotation that we do see is a remnant of the initial angular momentum, processed by several stages of stellar evolution. 


\section{Expectations from Scaling Arguments - Fast versus Slow}

One curiosity instantly arises in the study of white dwarfs rotation - that is, what should be considered a "fast" rotation velocity, and what is a "slow" rotation rate? As we will see below, measured white dwarf rotation rates seem to cluster around once per day. The literature contains examples of papers that talk about such rates as being fast, and others that refer to them as slow. This ambiguity stems from the two extreme cases of angular momentum redistribution that bracket what actually happens in stars. Assuming that local angular momentum (hereafter " $j$ ") is conserved through evolution results in an estimate of rotation at a rate of several hundred $\mathrm{km} \mathrm{s}^{-1}$. Alternatively, assuming that stars always rotate as solid bodies leads to an estimate of very long rotation periods (following loss of the extended AGB envelope). The view that even small magnetic fields can enforce solid-body rotation in all phases of evolution (i.e. Spruit 1998) leads directly to this sort of result.

Let's see more quantitatively what these two extreme cases imply. Assume that a $0.6 \mathrm{M}_{\odot}$ white dwarf star begins its life on the main sequence with a total mass of $3 \mathrm{M}_{\odot}$ and total angular momentum consistent with the Kraft relation (i.e. Kawaler 1987 and references therein). Thus the initial rotation period is approximately 10 hours.

If $j$ is retained throughout the evolution, internal differential rotation must develop, as parts of the star expand or shrink through evolution. By ignoring the rotational instabilities that should result from such angular velocity gradients, we can obtain an upper limit to the rotation rate of white dwarfs. The central $0.6 \mathrm{M}_{\odot}$ of a $3 \mathrm{M}_{\odot}$ main-sequence star collapse by a factor of about 20 by the time the star eventually reaches white dwarf dimensions. Thus it will spin up by approximately a factor of 400 , have a rotation period of about 100 seconds, and a surface equatorial rotation velocity of $680 \mathrm{~km} \mathrm{~s}^{-1}$. Under this assumption, then, anything slower than many hundreds of $\mathrm{km} \mathrm{s}^{-1}$ for a rotation velocity would be considered slow rotation.

If the white dwarf evolves from a lower mass progenitor, that the rotation velocity could be much lower even in this case. Stars of main-sequence spectral class of late $\mathrm{F}$ or later can lose up to $99 \%$ of their angular momentum before and on the main sequence. If they conserve $j$, their rotation rates as white dwarfs could be as small as about $10 \mathrm{~km} \mathrm{~s}^{-1}$. But, given the demographics of white dwarfs (a mean mass of about $0.6 \mathrm{M}_{\odot}$ and Population I kinematics) suggest that most that we see are derived from stars above the break in the Kraft curve, and therefore should have rotation velocities of hundreds of $\mathrm{km} \mathrm{s}^{-1}$ if they are described by the assumption of locally conserved angular momentum.

Next, consider a star that forever rotates as a solid body. When it reaches the AGB, its radius will have swelled to 90 times its main-sequence radius. It will then be rotating at a rate that is roughly 7500 times slower than its main-sequence rate, with nearly all of the angular momentum seated in the outer layers at large radius. The rotation period is $8.5 \mathrm{yr}$. Upon loss of the envelope and exposure of the central $0.6 \mathrm{M}_{\odot}$ white dwarf, the rotation period may shorten by a factor of 4 as the remnant contracts, leaving a white dwarf with a rotation period of about $2 \mathrm{yr}$ and a vanishingly small equatorial rotation velocity of about 1 meter per second. This assumption gives an upper limit to the rotation velocity, as stars must lose angular momentum as they lose mass 
on the AGB. Thus, under the assumption of perpetual solid-body rotation, any measurable rotation in a white dwarf should be considered fast rotation!

The fact that some white dwarfs are seen to rotate argues that some other mechanism is needed to be invoked to impart angular momentum to the remnant (e.g. Spruit 1998) in this extreme. Spruit (1998) points out that even small magnetic fields should enforce solid-body rotation in all phases of evolution and that they should show therefore no measurable rotation. The fact that they are indeed seen to rotate suggests a different origin entirely outside of the primordial angular momentum. He argues that some white dwarfs may in fact rotate because of asymmetric mass loss on the AGB phase, and during the final superwind phase. Though this is possible, we outline below another origin for the observed angular velocities in white dwarfs.

\section{Observations of White Dwarf Rotation}

Traditional measurements of rotation rates for stars - photometric variations from spots and rotational broadening of otherwise narrow absorption lines - fail for white dwarfs. White dwarfs are spotless stars - photometric time series do not show evidence of rotational modulation by spots. With very high gravities producing lines that are $10 \mathrm{~nm}$ wide or more, natural broadening mechanisms swamp rotational broadening - even for velocities close to breakup. However, discovery of sharp cores in the spectral lines of some DA white dwarfs, and of nonradial pulsations that are influenced in a predictable way by rotation, have produced a handful of useful (single) white dwarf rotation velocities. Magnetic white dwarfs, with magnetic fields in excess of $100 \mathrm{kG}$, show evidence of timevariability (and in some cases periodicity) of the magnetic features, providing a measurement of their rotation velocities. Given that such magnetic white dwarfs are rare (approximately $1 \%$ of white dwarfs) and that the magnetic fields can influence the rotational evolution, we do not discuss them further in this review - but see the excellent review by Wickramasinghe \& Ferrario (2000) for more on these fascinating objects.

\subsection{Spectroscopic Measurements of $v \sin i$ in White Dwarfs}

It was not until the early 1970s that Greenstein \& Petersen (1973) reported the detection of narrow cores within the hydrogen lines of DA white dwarfs. Modeling these narrow cores, via NLTE effects convolved with rotational broadening, allowed the possibility of measuring $v \sin i$. FIrst results using these cores, from Greenstein and Petersen (1973), Greenstein et al. (1977) and Pilachowski \& Milkey $(1984,1987)$ all showed rotation velocities at and below about $50 \mathrm{~km} \mathrm{~s}^{-1}$ - which was seen largely as slow rotation under the prevailing assumption of conservation of $j$. More recent high-resolution spectroscopy of white dwarfs by Heber et al. (1997) and Koester et al. (1998) produced more accurate measurements than the earlier work, but have not altered the conclusions drawn. Heber et al. (1997) report upper limits to rotation velocities to single non-magnetic white dwarfs. and Koester et al. (1998) report rotation velocities for only a handful of non-magnetic DA white dwarfs, including three ZZ Ceti stars.

Table 1 includes a representative sample of the upper limits and measurements of $v \sin i$ for white dwarfs. Note that except for the DAV (ZZ Ceti) stars, 
only GD140 shows a rotation velocity that is not consistent with zero, and that the uncertainties in the measurements are all of order $10 \mathrm{~km} \mathrm{~s}^{-1}$. This is difficult spectroscopy - high resolution spectra are needed, and accurate and reliable NLTE model atmospheres are essential for the analysis.

Table 1. Representative white dwarf rotation rates.

\begin{tabular}{|c|c|c|c|c|c|c|c|}
\hline Star & $M / \mathrm{M}_{\odot}$ & $T_{\text {eff }}$ & $\log g$ & $v\left[\mathrm{~km} \mathrm{~s}^{-1}\right]$ & $P_{\text {rot }}[\mathrm{d}]$ & type & Ref \\
\hline \multicolumn{8}{|c|}{ Spectroscopic determinations } \\
\hline 40 Eri B & 0.5 & 16500 & 7.86 & $\lesssim 8$ & $\gtrsim 0.09$ & DA & 1 \\
\hline LP 207-7 & 0.05 & 13600 & 7.76 & $\lesssim 18$ & $\gtrsim 0.04$ & $"$ & 1 \\
\hline G $148-7$ & 0.61 & 15500 & 7.97 & $\lesssim 12$ & $\gtrsim 0.061$ & $"$ & 1 \\
\hline HZ43 & 0.53 & 49000 & 7.70 & $\lesssim 29$ & $\gtrsim 0.025$ & $"$ & 1 \\
\hline G 1423-B2B & 0.52 & 14000 & 7.83 & $\$ 9$ & $z_{0.081}$ & & 1 \\
\hline LB 253 & - & 19200 & 7.8 & $20 \pm 20$ & 0.03 & $"$ & 3 \\
\hline WD $1337+70$ & - & 21000 & 8.0 & $30 \pm 20$ & 0.02 & $"$ & 3 \\
\hline GD 140 & - & 23000 & 8.0 & $60 \pm 10$ & 0.012 & $"$ & 3 \\
\hline W1346 & - & 21500 & 8.0 & $10 \pm 10$ & 0.06 & $"$ & 4 \\
\hline Grw +738031 & - & 15400 & 8.0 & $40 \pm 20$ & 0.015 & $"$ & 4 \\
\hline G29-38 & 0.6 & 11600 & 8.1 & $45 \pm 5$ & 0.014 & DAV & 2 \\
\hline GD165 & 0.6 & 11950 & 7.9 & $27 \pm 7$ & 0.024 & DAV & 2 \\
\hline L19-2 & 0.6 & 12200 & 8.0 & $38 \pm 3$ & 0.017 & DAV & 2 \\
\hline \multicolumn{8}{|c|}{ Asteroseismic determinations } \\
\hline G29-38 & 0.6 & 11600 & 8.1 & 0.55 & 1.35 & DAV & 5 \\
\hline GD165 & 0.6 & 11950 & 7.9 & 0.18 & 4.20 & $"$ & 6 \\
\hline L19-2 & 0.6 & 12200 & 8.0 & 0.63 & 1.10 & $"$ & 7 \\
\hline HS0507 & 0.6 & 12000 & 8.0 & 0.47 & 1.54 & $"$ & 8 \\
\hline GD 358 & 0.61 & 24000 & 8.0 & 0.606 & 1.20 & DBV & 9 \\
\hline PG $0122+200$ & 0.68 & 75500 & 7.5 & 0.455 & 1.60 & DOV & 10 \\
\hline PG $2131+066$ & 0.62 & 80000 & 7.5 & 3.487 & 0.21 & $"$ & 11 \\
\hline PG 1159-035 & 0.59 & 140000 & 7.5 & 0.527 & 1.38 & $"$ & 12 \\
\hline RXJ 2117 & 0.56 & 170000 & 6.0 & 0.627 & 1.16 & PNNV & 13 \\
\hline NGC 1501 & 0.55 & 81000 & 6.5 & 0.622 & 1.17 & & 14 \\
\hline
\end{tabular}

1. Heber et al. (1997); 2. Koester et al. (1998); 3. Pilachowski \& Milkey (1984); 4. Pilachowski \& Milkey (1987); 5. Kleinman et al. (1998); 6. Bergeron et al. (1993); 7. O'Donaghue \& Warner (1987); 8. Handler et al. (2002); 9. Winget et al. (1994); 10. O'Brien et al. (1998); 11. Kawaler et al. (1995); 12. Winget et al. (1991); 13. Vauclair et al. (2002); 14. Bond et al. (1996)

\subsection{Asteroseismology}

With few rotation velocities through spectroscopy, we must rely on a relatively new technique to provide data to constrain models of angular momentum evolution in highly evolved stars. This new technique exploits the observed nonradial $g$-mode pulsations of white dwarfs in several distinct bands in the H-R diagram. We see multimode nonradial pulsations in DA white dwarfs with effective temperatures at about $12,000 \mathrm{~K}$, in DB white dwarfs at about $25,000 \mathrm{~K}$, and in 
helium-poor C/O rich hot pre white dwarfs between $170,000 \mathrm{~K}$ and $75,000 \mathrm{~K}$. Comprehensive review of the many results for pulsating white dwarfs can be found in many volumes (two personal favorites are Kawaler 2000 and Kawaler 1996, but see also Fontaine, Brassard, Charpinet 2003). Other papers in this volume (i.e. Christiansen-Dalsgaard, and Matthews) describe the effect of slow rotation on the observed nonradial pulsation frequencies, so I only briefly review a basic asymptotic result as it has been applied to pulsating white dwarfs.

Nonradial modes of a given degree $\ell$ and radial overtone $n$ will have the same pulsation frequency, independent of $m$, in a spherically symmetric star. Rotation can break this degeneracy. For uniform rotation in white dwarfs that are pulsating in $g$-modes,

$$
\nu_{n \ell m}=\nu_{n \ell 0}+\frac{m}{P_{\text {rot }}}\left[1-\frac{1}{\ell(\ell+1)}\right],
$$

which is a limiting case where the pulsation motion in the horizontal direction is much larger than the vertical direction. So, if modes with all values of $m$ are excited, the pulsation modes should be split into triplets for $\ell=1$, into quintuplets for $\ell=2$, etc. Also, the splitting of these multiplets should be different for different values of $\ell$. In one star, PG 1159, Winget et al. (1991) show that these two effects are present: a sequence of triplet modes are split by equal amounts, and are nearly exactly 0.6 times the splitting seen in several quintuplets. The theory works quite well.

Rotational splitting has been seen in several white dwarfs (see Table 1). Asteroseismology clearly produces rotation frequencies of much higher precision (especially at smaller rotation rates) than is possible through spectroscopy. Oddly, those three DAV stars that have rotation determinations via spectroscopy show large discrepancies between the spectroscopic and asteroseismic values. Given the uncertainties in the spectroscopic values as mentioned above, and the multiple determination of rotational splitting within individual stars, the asteroseimsic values are probably more representative. We speculate that the broadening of the NLTE spectral line cores measured by Koester et al. (1998) may be due to some other photospheric motions related to the pulsation.

Is it possible that the interior of white dwarfs, where the pulsation frequencies are determined, rotates much slower than the surface? Kawaler, Sekii, \& Gough (1999), explored the effects of differential rotation within DOV and DBV white dwarfs. They concluded that the surface layers dominate the computed value of the rotational splitting in these stars. The cooler DAV stars are even more heavily influenced by the surface layers. Therefore the seismological rotation rates should be similar to the surface rotational broadening component of spectral lines. (Though Winget et al. (1994) suggested that the DBV GD358 showed evidence of differential rotation, Kawaler et al. (1999) showed that the variation in rotational splitting with $n$ could not be explained by a simple internal angular velocity gradient.)

The conclusion that we draw about measured rotation rates for white dwarfs, principally through asteroseismology, is that they rotate with periods of about once per day, with a range from a few hours to a few days. This is strikingly similar to the average rotation rate measured in magnetic white dwarfs, as reported by Wickramasinghe \& Ferrario (2000). So, white dwarfs rotate slowly (or is that quickly?). 


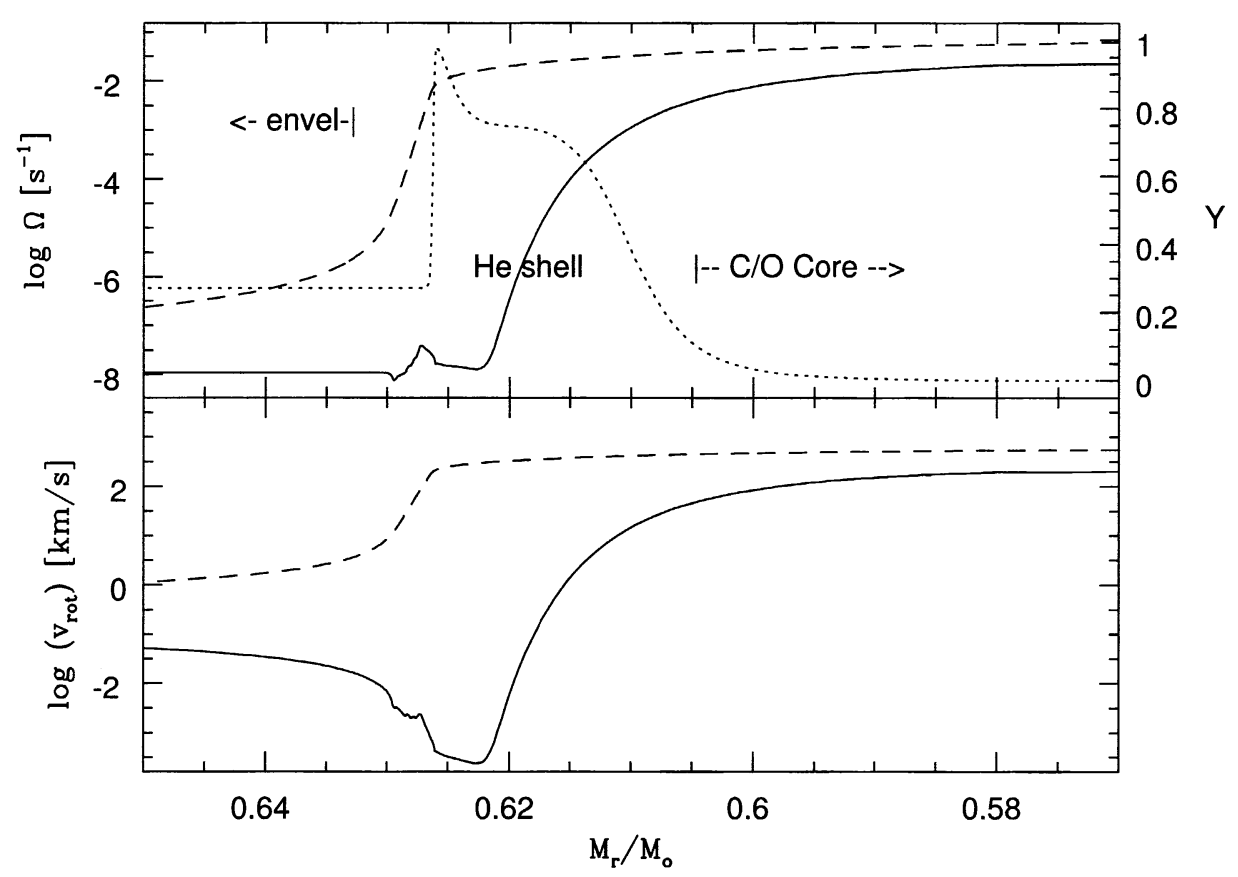

Figure 1. The core boundary within an ADB model, following a thermal pulse. The abscissa is $M_{r}$ with the center to the right. Top panel: angular velocity $\Omega$ (solid line); the dashed line shows $\Omega$ if one assumes constant $j$ everywhere throughout the evolution. The helium mass fraction (dotted line) has been influenced by recent convective shell burning during the thermal pulse. Bottom panel: $v_{\text {rot }}$ in the two limits.

\section{Evolutionary Models Using a Limiting Case for $j$ Redistribution}

With a bit of effort, evolutionary models that take us from the ZAMS through the AGB phase can make more specific predictions about what the rotation rate for white dwarfs might be. Through helioseismology, we now know that the interior of the Sun rotates as a solid body in the outer layers. This (along with "common wisdom") suggests that we should force convective regions within stellar models to rotate as solid bodies, with the total angular momentum within the convection zone as a conserved quantity. As the convection zone grows to incorporate more of the star, the angular momentum "dredged up" is distributed through the convection zone. For a growing surface convection zone, which is the dominant effect for AGB models, this results in a draining of angular momentum from inner regions to outer layers. A retreating convection zone releases the newly radiative regions to rotate with conserved local angular momentum.

To do this, we started with evolutionary sequences, begun on the ZAMS and evolved through several thermal pulses on the AGB (Kawaler et al. 2002). We then extracted the cores of these AGB models and evolved them into the white dwarf domain. Throughout the calculation, we kept track of angular momentum 


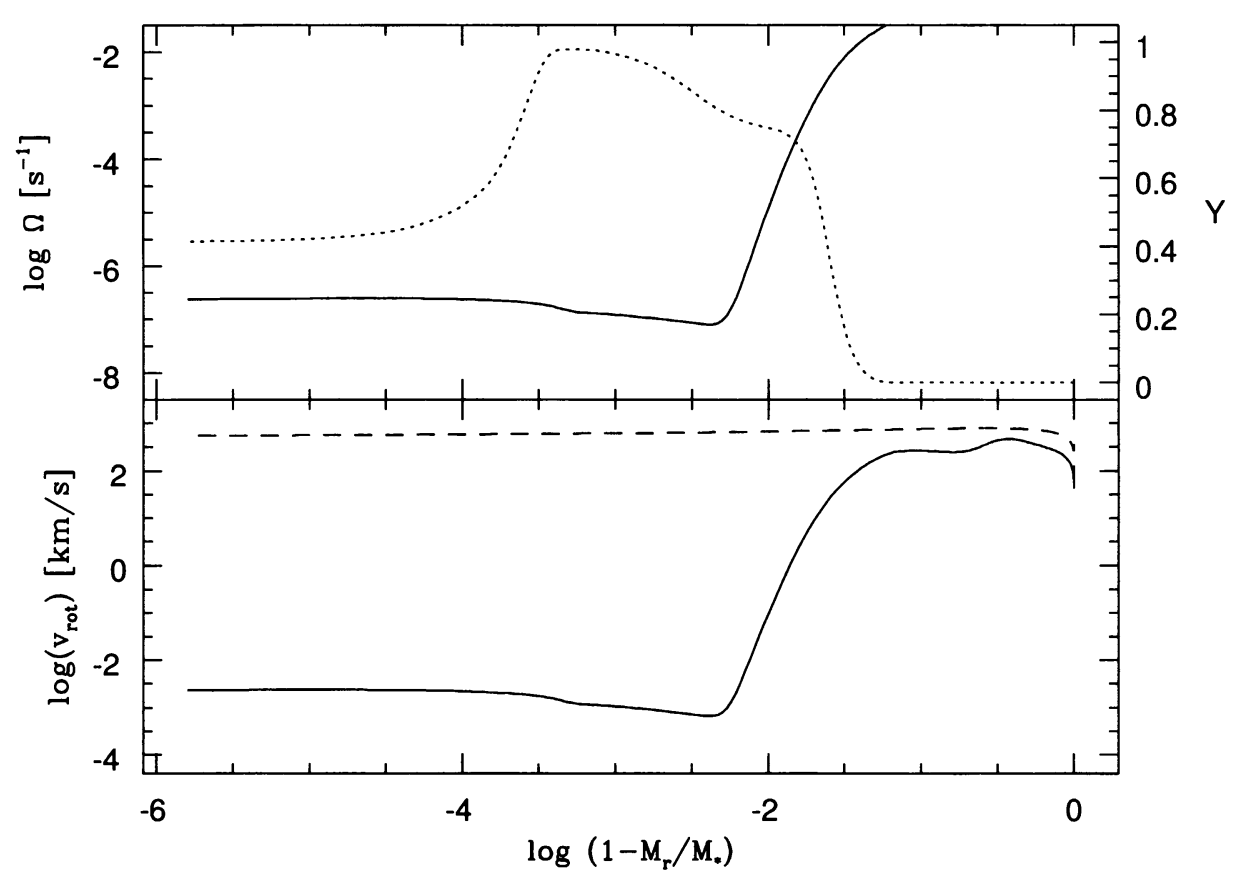

Figure 2. Rotation profile within a hot hydrogen-rich DA white dwarf model. Properties are plotted with respect to the log of the surface mass fraction, with the surface to the left and the center to the right. Lines are as described for Figure 1.

within the models using the prescription described above: conservation of $j$ in radiative regions, but solid body rotation within convective regions.

A sample "seed model" on the AGB is shown in Figure 1. This model is recovering from a recent thermal pulse. Convective helium burning has produced a marked plateau in the helium abundance profile at about $0.620 \mathrm{M}_{\odot}$ from the center. The transition zone from hydrogen to helium at $0.626 \mathrm{M}_{\odot}$ is the location of the recovering hydrogen burning shell, which lies just below the extensive convective envelope. The angular velocity profile through this region reflects the flat rotation curve in the convection zone. The small rotational velocity inversion at the hydrogen shell caused by its contraction that followed its release by the convective envelope, and the rise in angular velocity within and below the helium burning shell and into the compact $\mathrm{C} / \mathrm{O}$ core.

If a star such as this leaves the AGB at this stage, it could produce a hydrogen-rich planetary nebula (PN) central star should mass loss leave a layer of approximately $10^{-4} \mathrm{M}_{\odot}$ of hydrogen at the top of the core. The core would then contract to white dwarf dimensions, with the outer layers spinning up. The resulting DA white dwarf would then have a rotation profile resembling that shown in Figure 2, which is the $0.626 \mathrm{M}_{\odot}$ white dwarf remnant from the model shown in Figure 1. Figure 2 plots quantities versus the log of the surface mass fraction to expand features around the composition transition zones. 


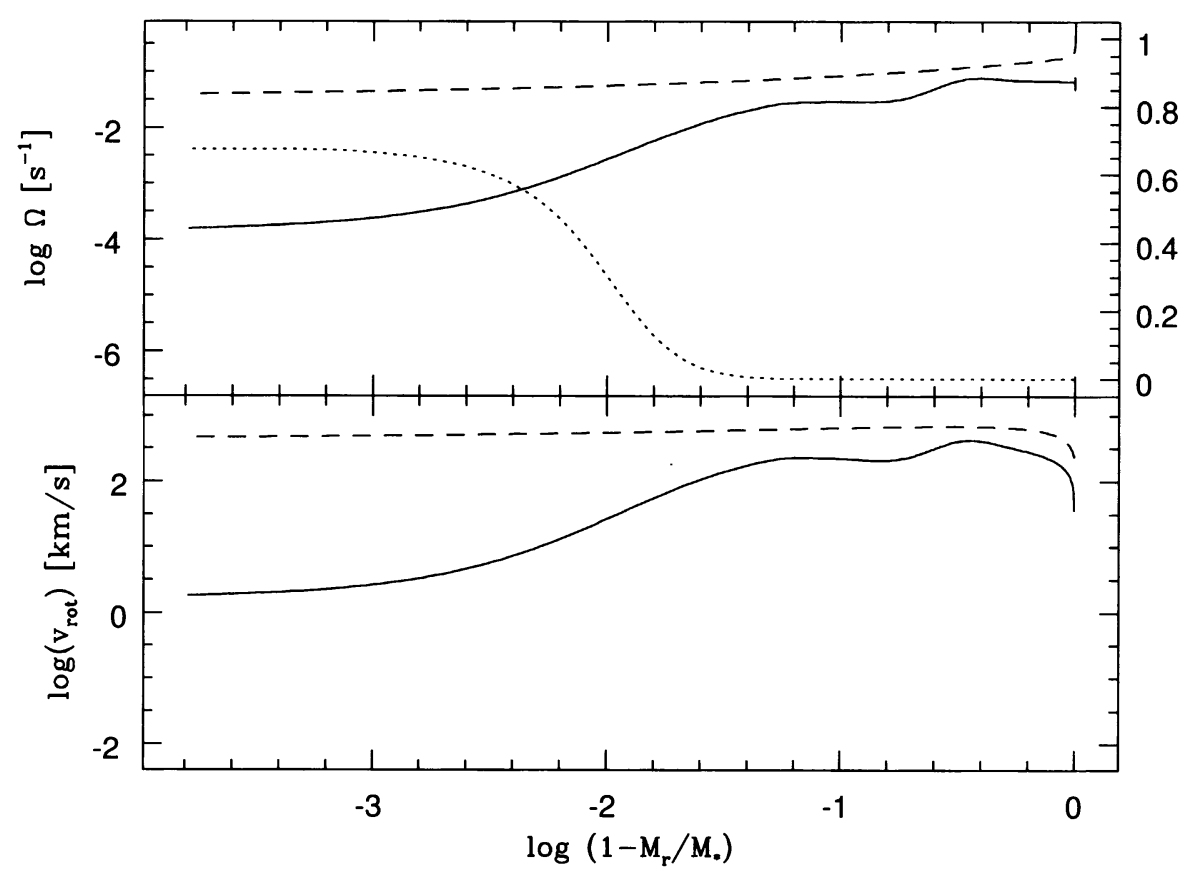

Figure 3. Same as Figure 2 but for a DB white dwarf model.

The situation for DB white dwarfs or PG 1159 stars is less desperate. Figures 1 and 2 , suggest that loss of mass into the plateau of the helium profile $\left(10^{-2}\right.$ $M *$ below the surface) will expose material with a much larger rotation velocity. Figure 3 shows a hot PG 1159 white dwarf model that represents the remnant of the model in Figure 1 should mass loss reach to the base of the plateau region. The rotation rate in the surface layers is quite close to the observed values.

These conclusions do depend on the initial angular momentum (and initial main sequence mass). We did not include any form of diffusive angular momentum transport, which could allow angular momentum to leak out of the core and spin up the surface layers. This would happen on time scales that are much longer than the time scales for evolution of hot white dwarfs. Typical angular momentum diffusion time scales are of order $10^{7} \mathrm{yr}$, which is 100 times longer than the time between thermal pulses; therefore, the angular momentum distribution within AGB stars is dominated by the convective envelope motion and convection within the thermally pulsing shells. Once a white dwarf is formed, takes much less than $10^{6} \mathrm{yr}$ to cross the H-R diagram and evolve down the top of the white dwarf cooling track.

Note that the observed rotation rates in pulsating white dwarfs, $\log v=$ -0.6 or $\log \Omega=-4.3$, are much faster than the model. Spectroscopic upper limits of $10 \mathrm{~km} \mathrm{~s}^{-1}$ are orders of magnitude larger than this model predicts.

Stars reach the DAV instability strip about $10^{8} \mathrm{yr}$ past the AGB; by then angular momentum diffusion may act to spin up the outer layers of DA white dwarfs. Langer et al. (1999) computed models up the AGB that included 
rotation and angular momentum redistribution. Though concentrating on rotationally induced mixing and its influence on nucleosynthesis, they extrapolate their results into the white dwarf phase to suggest that white dwarf rotation velocities of about $25 \mathrm{~km} \mathrm{~s}^{-1}$ might be expected. This conclusion does depend on the precise mass of the remnant (as indicated in our simpler models above), and is is clearly faster than seen in the pulsating DA white dwarfs.

\section{Conclusions, and Speculations}

Spectroscopic measurements of $v \sin i$ in single white dwarfs provide weak constraints on their rotation. Asteroseismology provides an accurate measurement, though only for those that are observed to pulsate. As the pulsators are representative of the class of stars, seismic rotation velocities can give some insight into the angular momentum evolution during the late stages of stellar evolution.

Reasonable assumptions about angular momentum transport within evolving stellar interiors points to several preliminary conclusions. Clearly, $j$ is drained from the outer core into the convective envelope on the AGB. As a result, DA white dwarfs are left with material with relatively low $j$, as their layers are repeatedly coupled to the stellar surface by convection. Thus they should (initially) rotate with periods much longer than one day. DB (and DO) white dwarfs have surfaces that were deeper than the deepest extent of the AGB envelope, and therefore retain higher $j$ - and should be rotating (initially) significantly faster than DA white dwarfs. Future work will concentrate on models of white dwarfs that include these angular velocity profiles, testing to see if the differential rotation can produce the observed changes in rotational splitting with overtone $n$ - in the manner described by Kawaler et al. (1999).

Finally, we point out that the rapid evolution along the AGB drives steep angular velocity gradients at the core/envelope boundary. Such steep gradients can perhaps drive a magnetic dynamo, and produce a shaping mechanism for planetary nebulae. This possibility has been investigated by several groups (see, for example, Blackman et al. 2001).

Acknowledgments. This work was supported, in part, by NASA ATP Grant NAG5-8352 to Iowa State University. Shelbi Hostler was instrumental in carrying out many of the calculations reported here, and Hugh Van Horn provided the stimulus and encouragement to look into this problem.

\section{References}

Bergeron, P., et al. (the WET collaboration) 1993, AJ 106, 1987

Blackman, E., Frank, A., Markiel, J.A., Thomas, J.H., \& Van Horn, H.M. 2001, Nature 409,485

Bond, H., et al. 1996, AJ 112, 2699

Fontaine, G., Brassard, P., Charpinet, S. 2003, in M. Thompson et al. (eds), Asteroseismology Across the HR Diagram, Ap\&SS 284, 257

Greenstein J.L., Boksenberg A., Carswell R., \& Shortridge K. 1977, ApJ 212, 186

Greenstein, J.L. \& Petersen, D.M. 1973, A\&A 29, 23

Handler, G., Romero-Colmenero, E., \& Montgomery, M. 2002, MNRAS 335, 399 
Heber, U., Napiwotski, R., \& Reid, I.N. 1997, A\&A 323, 819

Kawaler, S., Hostler, S., \& Burkett, J. 2002, in: R. Silvotti \& D. DiMartini (eds.), NATO Advance Research Workshop: The 13th European Workshop on White Dwarfs, (Dordrecht: Kluwer), in press

Kawaler, S. 2000, in: C. Ibanoglu (ed.), NATO Advanced Study Institute: Variable Stars as Astrophysical Tools, (Dordrecht: Kluwer), 511

Kawaler, S., Sekii, T., \& Gough, D. 1999, ApJ 516, 349

Kawaler, S. 1996, in: G. Meynet \& D. Schaerer (eds.), Stellar Remnants: Saas Fee Advanced Course 25, (Berlin: Springer), 1

Kawaler, S. et al. (the WET collaboration) 1995, ApJ 450, 350

Kawaler, S. 1987, PASP 99, 1322

Kleinman, S. et al. (the WET collaboration) 1998, ApJ 495, 424

Koester, D., Dreizler, S., Weidemann, V., \& Allard, N. 1998, A\&A 338, 612

Langer, N., Heger, A., Wellstein, S., \& Herwig, F. 1999, A\&A 346, L37

O'Brien, M.S. et al. (the WET collaboration) 1998, ApJ 495, 458

O'Donoghue, D. \& Warner, B. 1987, MNRAS 228, 949

Pilachowski, C. \& Milkey, R. 1984, PASP 96, 821

Pilachowski, C. \& Milkey, R. 1987, PASP 99, 836

Roxburgh, I.W. 1965, Zeitschrift für Astrophysik 62, 134

Spruit, H. 1998, A\&A 333, 603

Vauclair, G. et al. (the WET collaboration) 2002, A\&A 381, 122

Wickramasinghe, D. \& Ferrario, L. 2000, PASP 112, 873

Winget, D. et al. (the WET collaboration) 1991, ApJ 378, 326

Winget, D. et al. (the WET collaboration) 1994, ApJ 430, 839 